\title{
Interactive comment on "Characterisation of aerosol size properties from measurements of spectral optical depth: a global validation of the GRASP-AOD code using long-term AERONET data" by Benjamin Torres and David Fuertes
}

Anonymous Referee \#3

Received and published: 16 December 2020

\section{General comments:}

The aim of this paper is to show that the GRASP-AOD code has the potential to be used for large scale datasets either for aerosol climate studies or for near real time modeler needs. The validation based on 2.8 million GRASP-AOD retrievals using AERONET AOD observations from 30 sites during 20 years makes the work robust enough to reach appropriate conclusions. The paper is to long taking into account the methodology used, the results and the prior knowledge published about this type of AOD 
published, including the new considerations used that can improve this type of AOD inversion codes (comparative and differences with other papers already published). The paper is well written and into the scope of AMT. I recommend the publication of this paper, but there are some issues should be addressed prior to publication. The Editor will judge.

The AOD inversion codes have used in different papers from many years. These type of inversion codes are based on the aerosol scattering equation that express the dependence of the spectral variation of $A O D$ on the aerosol size distribution, and also depend of the Qext parameters (particle extinction efficiency factors), which in turn depend on the wavelength, the refractive index and particle radius. As example, King et al. (1978) already pointed out that the definition of the particle radius interval on which the inversion method can be correctly used, and the assumption of realistic refractive index values are the most crucial points in any rigorous application of inversion methods applied to spectral series of the AOD. On the other hand, the independent information content on the optical characteristics of columnar aerosols is contained primarily in the particle radius interval from 0.1 to 2 microms, approximately, for AOD measurements covering spectral range $340-1020 \mathrm{~nm}$. On the other hand, the iterative procedures modified the radius interval within the prescribed ranges, and the best results were obtained for reduced radius range. In this sense, with this type of codes the results are limited to the accumulation mode. On the other hand, some AOD inversion algorithms use a single refractive index, while the true is dependent on wavelength. The assumption of an a priori defined refractive index in the AOD inversion procedures may lead to very different derive size distributions, but other authors (e.g., Yamamoto and Tanaka, 1969; King et al., 1978; González and Ogren, 1996) show that the shape of the retrieved aerosol size distribution is not substantially altered as a result of using such assumptions. In this sense, this paper should take into account previous work and show the improvements that can be made. Taking into account previous results, obviously these type of inversion algorithms would not work well for coarse particle modes just considering only the AOD spectral values. Spectral aureole data (sky radiances) 
-Lines 85-95. To motive the importance of this work, the authors comments that many AERONET sites are plagued by several months of partial cloudiness (no sky radiance measurements) ... but later they use climatological values for refractive index and information about radius modes. How it is possible for this type of AERONET stations, and how representative are these values? also for future applications to night measurements. The columnar aerosol properties change from day to night, depend on sources, the air masses transport, the planetary boundary layer high ... Also, a study of the GRASP-AOD sensitivity to the refractive index is needed.

-Line 185. The GRASP-AOD code assumes the refractive index as known. Which one has been chosen for each AERONET station and aerosol type? Can be Included in Table-1? On the other hand, the aerosol type selected for each station (Table 1) can be the more frequent (climatology), but not all ways are the same. As example, the Saharan dust outbreaks. How these facts affect the inversion products?

Lines 190-195. If the refractive indices are assumed, what happens, as example, with stations where there are many clouds and cannot be computed with the sky radiance data? There are no data? Do you use the climatological value? How much data have you used to obtain this climatological value, and how is it distributed throughout the year? In order to these results will be realistic, an extensive database should be available and the appropriate refractive index value used for each atmospheric condition. The purpose of this work is to show that the GRASP-AOD application has the potential to be used for large scale datasets.

Lines 480-525. Obviously, the algorithm does not work well for coarse particle mode just taking into account only the AOD spectral parameters and a climatological value of the refractive index. But we already knew these results from the papers published related with these type of inversion codes. The sky radiance data is needed to achieve

Interactive comment
Printer-friendly version

Discussion paper 
good results in coarse mode. I think this section should be shortened or removed from the paper. Also, the last sentence of the abstract is a well-known result and it is not AMTD new.

Lines 200-225. The criteria are based mostly on analyst's experience. The authors show "Due to the low sensitivity of GRASP-AOD to the shape of the modes... we have used strong a priori constraints on the actual values for the standard deviation of both modes... in practice, their values are very similar to the given initial guess values". On the other hand, in Line 340 the authors show: "The larger uncertainties observed for Solar Village compared to GSFC can be extrapolated to all sites with coarse mode predominance with respect to the sites with fine mode predominance", and the following lines. Taking into account the papers published so far, it is clear that this methodology can only be applied to places where the fine mode predominates.

In my opinion, this work should be drastically reduced, showing only those aspects that can improve the results of the works already published. On the other hand, the usefulness of using climatological values in the a priori assumptions should be better discussed, taking into account the sensitivity of the GRASP-AOD model to the parameters.

Interactive comment on Atmos. Meas. Tech. Discuss., doi:10.5194/amt-2020-426, 2020.

Interactive comment

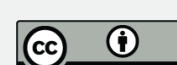

